\title{
ABCB1 c.3435C > T and EPHX1 c.416A > G polymorphisms influence plasma carbamazepine concentration, metabolism and pharmacoresistance in epileptic patients
}

\author{
Ming-Liang Zhang ${ }^{1}$, Fang-Zhou Liu ${ }^{1}$, Xiao-Long Chen ${ }^{1}$, Wei-Xia Li ${ }^{1}$, Xiao-Yan Wang ${ }^{1}$, Hui \\ Zhang ${ }^{1}$, Pan-Pan Chen ${ }^{1}$, Lu Niu ${ }^{1}$, Yan Wang ${ }^{1}$, Wen-Hui Jia ${ }^{1}$, and Jin-Fa Tang ${ }^{2}$ \\ ${ }^{1}$ Affiliation not available \\ ${ }^{2}$ The First Affiliated Hospital of Henan University of Chinese Medicine
}

May 19, 2020

\begin{abstract}
Background: ABCB1, EPHX1 and SCN1A gene polymorphisms have been reported play important roles in individual variability carbamazepine (CBZ) metabolism and resistance, but the result of that association still remains controversial. Objective: To clarify the associations among ABCB1, EPHX1 and SCN1A gene polymorphisms and CBZ metabolism and resistance. Methods: The PubMed, EMBASE, Cochrane library, Chinese National Knowledge Infrastructure, Chinese Science and Technique Journals Database, China Biology medicine disc and Wan Fang Database were searched for appropriate studies up to April 2020. Results: A total of 18 studies involving 3293 related epilepsy patients were included. ABCB1 c.3435C $>$ T polymorphism was significantly associated with adjusted concentrations of CBZ (CC vs. CT, $\mathrm{P}=0.004)$, and EPHX c.416A $>\mathrm{G}$ polymorphism was significantly associated with carbamazepine-10, 11-trans dihydrodiol (CBZD) (AA vs. GG, $\mathrm{P}=0.045 ; \mathrm{AG}$ vs. $\mathrm{GG}, \mathrm{P}=0.010$ ). Furthermore, ABCB1 c.3435C $>$ T polymorphism was also observed to be significantly influenced CBZ resistance (CT vs TT, $\mathrm{P}=0.01 ; \mathrm{CC}+\mathrm{CT}$ vs $\mathrm{TT}, \mathrm{P}=0.006)$. Conclusion: $\mathrm{ABCB} 1$ c. $3435 \mathrm{C}>\mathrm{T}$ polymorphism may affect the CBZ metabolism and resistance, EPHX1 c.416A $>$ G polymorphism may only affect CBZ metabolism. These findings provided further evidence for individualized therapy of epilepsy patients in clinics. Nevertheless further large studies are still warranted to provide conclusive evidences.
\end{abstract}

\section{Introduction}

Carbamazepine (CBZ), prescribed as a first-line treatment for focal or generalized tonic-clonic epileptic seizures, is diverse from individuals among CBZ daily maintenance doses, plasma concentrations, and treatment efficacy [1]. The difference of pharmacodynamics and pharmacokinetics of CBZ varied from environmental factors co-medication to genetic variation [2], among which genetic variation of drug-resistance and drug-metabolizing enzymes were found play critical roles in CBZ therapy for epileptics $[3,4]$.

CBZ, catalyzed by cytochrome P450 3A4 (CYP3A4), can generate a bioactive metabolite carbamazepine10,11-epoxide (CBZE) of which possess a strongly antiepileptic activity [5]. Subsequently, CBZE inactivated to carbamazepine-10,11-transdihydrodiol (CBZD) by microsomal epoxide hydrolase which is encoded by microsomal epoxide hydrolase (EPHX1 ) [6]. Meanwhile, the genetic variants of c.337T>C (rs1051740) and c.416A>G (rs2234922) in EPHX1 were found associated with the pharmacokinetics and pharmacodynamics of CBZ [7, 8]. P-glycoprotein (P-gp), producted by ATP binding cassette transporter, subfamily B, member $1(A B C B 1)$ or multidrug resistance 1 gene $(M D R 1)$, is a highly reported drug efflux transporter as CBZ substrates [9-11]. As a highly polymorphic drug-resistance gene, the $A B C B 1$ polymorphisms of c.3435C $>T$ (rs1045642) in exon 26,c.1236C>T (rs1128503) in exon 12 and c.2677G>T/A (rs2032582) in exon 21 have been extensively studied can influence the extent of expression and transport activities of P-gp [11]. The 
c.3184A>G (rs2298771) and IVS5-91G>A (rs381278) polymorphisms in SCN1A alter the sodium channels structure and functions relationship with $\mathrm{CBZ}$, ultimately rendering them insensitive to the blocking effect of CBZ, and SCN1A polymorphisms has been found to be associated with CBZ-resistant epilepsy [12-14].

In 2019, Gui-Xin Zhao et al. [15] performed a meta-analysis revealed an association between EPHX1 c.337T>C and c. 416A>G polymorphisms and CBZ metabolism in epilepsy with 7 articles were included, but the $\mathrm{CDR}_{\mathrm{CBZ}}$ data units were inconsistent in the Chin-Chuan Hung [16] and Chun-Lai Ma [17] studies were also directly meta-analyzed, which will bring a huge deviation in the outcome. Meanwhile, the relationship between $A B C B 1$ (C.3435C>T, c.2677G $>T / A$ and c.1236C>T), EPHX1 (c.337T>Cand c.416A>G) polymorphisms and $\mathrm{CBZ}$ resistance still remains contradictory. In order to realize the individualized treatment of CBZ in epileptic patients, we performed a systematic meta-analysis to evaluate the effects of $A B C B 1$ (C.3435C>T, c.2677G $>T / A$ and c.1236C $>T$ ) and $E P H X 1$ (c.337T>C and c.416A>G) polymorphisms on CBZ metabolism and resistance.

\section{Methods}

\subsection{Search and inclusion/exclusion criteria}

The present systematic review was based on with the PRISMA statement principles [18]. The PubMed, EMBASE, Cochrane library, Chinese National Knowledge Infrastructure, Chinese Science and Technique Journals Database, China Biology medicine disc and Wan Fang Database were searched up to 30 April 2020 using the boolean combination of the following key terms: "Carbamazepine" OR " $A B C B 1$ " OR "MDR1" OR "EPHX1" OR "SCN1A" AND "polymorphism". Eligible studies were required to meet the following inclusion criteria: (i) case-control studies; (ii) investigating the association of $A B C B 1$ polymorphisms and or EPHX1 polymorphisms and plasma concentration or resistance of CBZ in epileptic patients; (iii) reporting sufficient data for estimating an odds ratio (OR) for the association with the gene variants of interest; (iv) each patient was administrated with CBZ monotherapy and not receiving pharmacological treatment for other pathologies; (v) including at least 3 studies for each polymorphism to allow calculation of publication bias between studies.

Exclusion criteria: (i) review articles and editorials; (ii) duplication of previous publications; (iii) not human studies; (iv) not English or Chinese articles. All potentially relevant studies retrieved in the initial screening step were then read in their entirety to assess appropriateness for inclusion in the systematic review. The reference lists of all included studies and relevant reviews were also checked to identify additional studies missed from the initial electronic search. The corresponding authors were contacted by e-mail when relevant data could not be extracted from the original paper. Studies were excluded if the corresponding author did not answer to the e-mail or was unable to provide the requested data. If two or more studies shared part of the same patient population, the one with the larger sample size was included. All studies were independently analysed by two reviewers and any discrepancies in data extraction were resolved through consensus.

\subsection{Data extraction and quality assessment}

Information was carefully extracted from all eligible publications by two independent authors, including author's name publication date, country, sample size, plasma concentration and adjusted serum concentration of $\mathrm{CDR}_{\mathrm{CBZ}}, \mathrm{CDR}_{\mathrm{CBZE}}$ and $\mathrm{CDR}_{\mathrm{CBZD}}, \mathrm{CDR}_{\mathrm{CBZE}}: \mathrm{CDR}_{\mathrm{CBZ}}$ and $\mathrm{CDR}_{\mathrm{CBZD}}: \mathrm{CDR}_{\mathrm{CBZE}}$ ratios, genotypes distribution, drug resistant patients (defined as no change or less than $50 \%$ reduction in seizure frequency for at least 1 year during treatment with CBZ and drug responsive patients defined as seizure-free or a $50 \%$ or greater reduction in seizure frequency for at least 1 year during treatment with CBZ) [8, 19]. If diverse results were generated the disagreement was resolved by discussion between the two authors or by asking

a third investigator. The authors of the studies were contacted for additional data when necessary and applicable.

\subsection{Assessment of methodological quality}

We used the Newcastle-Ottawa scale (NOS) [20] for assessing the quality of the included studies in this 
meta-analysis. Using this scale system, each included study was judged on three broad perspectives: the selection of the study groups, the comparability of the groups, and the ascertainment of outcome of interest. High-quality studies were identified with a NOS score of five or more. Whereas those with less than a score of five were considered as low-quality studies.

\subsection{Statistical analysis}

We conducted the review using the Stata 12.0 and RevMan 5.3 software. Comparisons of the dichotomous variables were performed using odds ratio (OR) with $95 \%$ confidence interval (CI). For continuous variables, the standard mean differences (Std. MD) with $95 \%$ CI were used. Z-test was performed to determine the statistical significance of results with statistical significance defined as $P<0.05$. Heterogeneity between articles was assessed by Cochrane's Q-test. Data with low heterogeneity $\left(P[?] 0.10\right.$ and $\left.I^{2}<50 \%\right)$ were analyzed by a fixed-effects model while a random-effect model was used for data with high heterogeneity $\left(P<0.10\right.$ and $\left.I^{2}>50 \%\right)$. To explore sources of heterogeneity the leave-one-out sensitivity analysis was also conducted. Furthermore, a funnel plot was used to evaluate the publication bias when applicable.

\section{Results}

\subsection{Characteristics of the identified studies}

The search criteria on PubMed, Web of Knowledge and Cochrane databases yielded 932 hits of which 324 were duplicates. After removal of additional 590 records (including one [21] of the two articles [21, 22] with the same sample published in different journals was excluded), 13 studies of CBZ resistance $[7,8,13,14$, 22-30] and 8 studies of CBZ metabolism [7, 8, 17, 25, 29, 31-33] were included in the systematic review (Fig. 1). All participants in the studies were epilepsy patients and were treated with CBZ monotherapy at a stable maintenance dose. The characteristics of included studies are presented in Table 1 and 2. All the patients were treated with CBZ in a dose range of $400-1200 \mathrm{mg} /$ day. The NOS scores of the included studies ranged from 5 to 8 based on the NOS evaluation system, indicating a relatively high quality of included studies. Furthermore, a test of funnel plot was not conducted due to the insufficient number of studies in the present study.

\subsection{Data Analysis}

Table 3 and 4 summarizes the main results of this meta-analysis. The pooled ORs were computed for the allelic contrast, recessive, homozygotes and dominant models.

\subsubsection{Associations between $A B C B 1$ (c.3435C>T), EPHX1 (c.337T>C and c.416A>G), $S C N 1 A$ (c.3184A $>G$ and $I V S 5-91 G>A$ ) polymorphisms and CBZ metabolism.}

For $A B C B 1$ c.3435C>T polymorphism, a total of 714 epilepsy patients treated by CBZ in 4 studies [8, $29,31,33]$ were included, and a statistically significant association was found in the (CC vs CT) model for the adjusted concentrations of $\operatorname{CDR}_{\mathrm{CBZ}}(O R=0.251$ (95\% CI: 0.081 0.42), $P=0.004)$ (Table 3) (Fig. 2). For $A B C B 1$ c. $2677 G>T / A$ polymorphism, a total of 569 epilepsy patients treated by CBZ in 3 studies were included, and a statistically significant association was found in the ( $G G$ vs $G A$ ) model for the adjusted concentrations of $\mathrm{CDR}_{\mathrm{CBZ}}(O R=-0.366$ (95\% CI: -0.697 -0.036), $P=0.030)$ with high heterogeneity $\left(\mathrm{I}^{2}=64.2 \%\right)$ (Table 3). There is no significant association between other genetic models in $A B C B 1$ c.3435C>T and $A B C B 1 \quad$ c.2677G $>T / A[29,31,33]$ (3 studies including 569 epilepsy patients) and the whole genetic models in $c .1236 C>T$ [29, 31-33] (4 studies including 659 epilepsy patients) polymorphisms on the adjusted concentrations of $\mathrm{CDR}_{\mathrm{CBZ}}$ (Table 3 ).

For EPHX1 c.416A>G polymorphism, the adjusted concentrations of $\mathrm{CDR}_{\mathrm{CBZ}}$, $\mathrm{CDR}_{\mathrm{CBZE}}$ and $\mathrm{CDR}_{\mathrm{CBZD}}$ were reported in $4[7,8,25,31], 3[7,8,31]$ and $3[7,8,31]$ articles, respectively. A total of 646 epilepsy patients treated by CBZ in 3 studies were included with statistically significant association between ( $A A$ vs $G G),(A G$ vs $G G)$ models in EPHX1 c.416A>Gpolymorphisms and $\mathrm{CDR}_{\mathrm{CBZD}}(O R=0.483$ (95\% CI: $0.011^{\sim}$ 0.956), $P=0.045 ; O R=0.682$ (95\% CI: 0.163〜1.201), $P=0.010$, respectively) (Table 3) (Fig. 3). 
No association was observed between other genetic models of $E P H X 1$ c. $416 A>G$ polymorphism and on the adjusted concentrations of $\mathrm{CDR}_{\mathrm{CBZ}}, \mathrm{CDR}_{\mathrm{CBZE}}, \mathrm{CDR}_{\mathrm{CBZD}}$, respectively (Table 3 ).

For EPHX1 c.337T>C polymorphisms, the adjusted concentrations of $\mathrm{CDR}_{\mathrm{CBZ}}, \mathrm{CDR}_{\mathrm{CBZE}}, \mathrm{CDR}_{\mathrm{CBZD}}$, $\mathrm{CDR}_{\mathrm{CBZE}}: \mathrm{CDR}_{\mathrm{CBZ}}$ and $\mathrm{CDR}_{\mathrm{CBZD}}$ : $\mathrm{CDR}_{\mathrm{CBZE}}$ including 812 epilepsy patients were reported in $4[7$, $8,25,31], 3[7,8,31], 3[7,8,31], 3[7,8,17]$ and $3[7,8,31]$ articles, respectively. A statistically significant association was found in the (TT vs $C C$ ) model for the adjusted concentrations of CDR CBZE $_{\text {. }}$ $(O R=-0.446$ (95\% CI: -0.811 -0.0.081), $P=0.004)$ with high heterogeneity ( $\left.\mathrm{I}^{2}=98.9 \%\right)$ (Table 3). No significant association among other genetic models of $E P H X 1$ c.337T $>C$ polymorphism and on the adjusted concentrations of $\mathrm{CDR}_{\mathrm{CBZ}}, \mathrm{CDR}_{\mathrm{CBZE}}, \mathrm{CDR}_{\mathrm{CBZD}}, \mathrm{CDR}_{\mathrm{CBZE}}: \mathrm{CDR}_{\mathrm{CBZ}}$ and $\mathrm{CDR}_{\mathrm{CBZD}}: \mathrm{CDR}_{\mathrm{CBZE}}$ were found (Table 3).

3.2.2 Associations between $A B C B 1$ (c.3435C>T), EPHX1 (c.337T>C and c.416A>G), SCN1A (c.3184A>G and IVS5-91G>A) polymorphisms and CBZ resistance.

For $A B C B 1$ c.3435C $>T$ polymorphism, a total of $344 \mathrm{CBZ}$ resistance and $458 \mathrm{CBZ}$ responsive in 5 studies $[8,22,27,29,30]$ were included in this meta-analysis. A statistically significant association was found in the (CT vs TT) and $(C C+C T$ vs TT) model $(O R=1.603$ (95\% CI: 1.119 2.298), $P=0.010 ; O R=1.61$ (95\% CI: 1.148 2.258), $P=0.006$, respectively) (Table 4) (Fig. 4). There is no associations between other genetic models in $A B C B 1$ C.3435C $>T$ polymorphism and CBZ resistance (Table 4).

For EPHX1 c.337T $>C$ and $c .416 A>G$ polymorphisms, a total of $113 \mathrm{CBZ}$ resistance and $233 \mathrm{CBZ}$ responsive in 3 studies $[7,8,25]$ were included in this meta-analysis with no associations in any genetic models of $E P H X 1$ c.337T>C and c.416A $>G$ polymorphisms for CBZ resistance. The same results were also observed in all genetic models $S C N 1 A$ c.3184A>G $[8,13,24,26]$ (4 studies including $430 \mathrm{CBZ}$ resistance and $597 \mathrm{CBZ}$ responsive) and IVS5-91G>A[8, 14, 23, 24, 26, 28] (6 studies including $612 \mathrm{CBZ}$ resistance and $648 \mathrm{CBZ}$ responsive) (Table 4).

\subsection{Heterogeneity and publication bias}

Egger's test showed no evidence of publication bias in the studies analysed except $A B C B 1 \quad$ c.3435C>T polymorphism in the $(C C$ vs $C T+T T)$ and $(C C+C T$ vs $T T)$ model. The distribution of the genotype in the CBZ responsive group of each study was consistent with HWE except 2 studies [22, 29] for $A B C B 1$ c.3435C $>T$ and 1 study [7] for EPHX1 c.337T>C and $c .416 A>G$. The heterogeneity and publication bias of included studies on each gene polymorphism are presented in Table 2. Considering that significant heterogeneity was identified in overall studies and the ineligible studies for HWE, we performed a sensitivity analysis by repetitive calculation of the combined OR estimate and a heterogeneity test after removing one study at a time. However, while remove 1 study [22] in the (CC vs TT) model of $A B C B 1$ c.3435C>T which is not conformed with HWE, the results of association changed with significant difference (OR $=1.781$ (95\% CI: 1.166 2.722), $P=0.008$ ) (Table 4).

\section{Discussion}

As a clinical first-line antiepileptic drug, CBZ has a relatively narrow therapeutic range $(4-12 \mu \mathrm{g} / \mathrm{mL})$ and wide inter-individual variability [34]. Genetic variants in $A B C B 1, E P H X 1, S C N 1 A$ genes are wellknown to influence the individual CBZ daily maintenance doses, metabolism and resistance for epileptic patients. Thus, clarify the role of $A B C B 1, E P H X 1, S C N 1 A$ genetic polymorphisms in pharmacokinetics and pharmacodynamics of CBZ will be helpful for the effective clinical individualized treatment of epilepsy.

The $A B C B 1$ (c.3435C>T, c.2677G $>T / A$ and c.1236C>T) in exon 26 closely related with the altered expression levels of $\mathrm{P}$-gp, range of CBZ resistance in epileptics. In the meta-analysis, c.3435C $>T$ and $c .2677 G>T / A$ polymorphisms were found to be associated with adjusted concentrations of CBZ. For the high heterogeneity in $c .2677 G>T / A$, we did not conduct subgroup analysis or sensitivity analysis limited by only 3 articles included. In order to seek the mechanism of $c .3435 C>T$ on the clinical effect of CBZ in epilepsy patients, we further analyzed whether $c .3435 C>T$ polymorphism change the clinical efficacy by influencing CBZ resistance, and found that patients with the $c .3435 C>T$ TT genotype exhibited lower CDZ resistance compared 
with $C T$ or $(C T+C C)$ genotype, Hongmei Meng's study [29] also supported it. This might attributable to the $C C$ genotype of $c .3435 C>T$ enhancing the expression of P-gp and excessive CBZ efflux across the bloodbrain barrier (BBB) resulting in drug resistance in epileptics. However, the included studies of Armond Daci [8] and Gulay Oner Ozgon's [30] results unsupported it in Kosovar Albanian and Turkey epileptic patients. This phenomenon may be attribute to ethnic differences, but we did not conduct racial analysis limited number of literatures included. Overall, our results confirmed c.3435C $>T$ polymorphism may influence the clinical efficacy via regulating CBZ resistance followed by adjusting serum concentration of CBZ although more high-quality original studies are still required.

As an active metabolites of CBZ, CBZ-10,11-epoxide (CBZE) is further transformed to an inactive metabolite, CBZ-10,11-diol (CBZD) via microsomal epoxide hydrolase ( $\mathrm{mEH}$ ) which encoded by EPHX1 [35, 36]. Thus, EPHX1 (c.337T>C and c.416A>G)mutation may affect CBZ pharmocokinetics and pharmacodynamics by changing the function of $\mathrm{mEH}[36,37]$. Our study found that the patients with $A A$ genotype in c. $416 A>G$ has lower level of $\mathrm{CDR}_{\mathrm{CBZD}}$, prompting that CBZ metabolism may be inhibited by the $c .416 A>G$ variant [37], which has been demonstrated to decrease $\mathrm{mEH}$ activity in Yukiko Nakajima's study [38]. However, no association between any genetic models of EPHX1 c.416A>G polymorphism and CBZ resistance was found, which supported by Wenting Yun et al. study [25]. In addition, we abandoned high heterogeneity data of $\mathrm{CDR}_{\mathrm{CBZE}}$ (for c.337T>C) and the instability data of CDR $\mathrm{CBZD}_{\mathrm{D}}: \mathrm{CDR}_{\mathrm{CBZE}}$ ratio (for $c .416 A>G$ ) limited by 2 articles with small samples. Furthermore, no association was found between EPHX1 c.416A>G and CBZ resistance in our present study. Therefore, EPHX1 c.416A>G associated with CBZ metabolism and resistance need to be further studied in the larger cohorts of CBZ for epilepsy.

IVS5-91G>A located at 5 splice donor site of $S C N 1 A$ exon $5 \mathrm{~N}$, plays a crucial role in epilepsy by encoding the S4 transmembrane segment or voltage sensor in the first domain of the Nav1.1 protein and initiating action potentials in neurons in different parts of the mammalian brain [39, 40]. Whilec.3184A>G polymorphism in $S C N 1 A$ may influence the structural and functional properties of sodium channels and further impact treatment of CBZ for epilepsy via converting threonine to alanine [24]. It was noteworthy that, no association was found based on the present included studies containing Chinese, Japanese, Serbia, and Pakistan epilepsy patients among SCN1A (c.3184A>G and IVS5-91G>A) and CBZ resistance, which cannot be further supported by CBZ metabolism for small numbers of literatures and samples included. This may be due to the lack of data for further evaluation differences in the geographical distribution of the subjects or environmental factors [41]. Consequently, further investigations based on more well-designed experiments and larger sample size should be performed to verify the effects of $S C N 1 A$ (c.3184A>G and IVS5-91G>A) on CBZ resistance and metabolism.

This meta-analysis first comprehensively collected the existing studies and reviewed the relationships among $A B C B 1$ (c.3435C>T), EPHX1 (c.337T>C and c.416A>G), SCN1A (c.3184A>G and IVS5-91G>A) polymorphisms and CBZ metabolisms and resistance. Compared with precious meta-analysis [15], the present study not only evaluated the associations between the $A B C B 1$ (c.3435C>T), EPHX1 (c.337T>C and c.416A>G), SCN1A (c.3184A>G and IVS5-91G>A) polymorphisms and drug-resistance risk, but also clarified the effect of corresponding genes on CBZ metabolism in epileptics. Consequently, our data suggest that $A B C B 1$ c.3435C>T and EPHX1 c.416A>G polymorphisms could influence inter-individual variability of $\mathrm{CBZ}$ metabolism and resistance.

The inconsistencies in the studies may be explained by some phenomena. Firstly, only English and Chinese studies were included, many literatures in other languages were ignored which may lead to the existence of bias. Secondly, the small sample size may result in false-positive problem and weaken the statistical power. Thirdly, the optimal dosage of CBZ may be influenced by a variety of confounding factors such as age, sex, ethnicity, and the interaction between gene mutations, but the lack of original data limited our ability to further assess these factors. Fourthly, lack of some important raw data limits the further evaluation of other indicators, such as the therapeutic effect of concentration-time curves on metabolic clearance and adverse events. Furthermore, based on Egger's test, we detected a small publication bias which might be caused by unpublished data, because negative studies were less likely to be published and included in 
journals and computerized database, and only studies indexed by the selected databases were included, this results in a potential overestimation of effect sizes. Finally, different doses and treatment durations were used in the included studies, which may affect the observable effect of pharmacokinetic genes. These caveats should be considered and overcome in future large sample and multiracial studies. In spite of above potential limitations, our meta-analysis collected the most recently published data on the association between $A B C B 1$, EPHX1 gene polymorphisms and CBZ metabolism and resistance.

\section{Conclusion}

Our results showed that $A B C B 1$ c.3435C $>T$ polymorphism was associated with adjusted concentrations of CBZ and drug-resistance, and EPHX1 c.416A>G polymorphism was related to decrease CBZD concentration, which confirmed important effects of $A B C B 1 \quad c .3435 C>T$ and $E P H X 1 \quad c .416 A>G$ gene on pharmacokinetics and pharmacodynamics of CBZ and would help improve individualized therapy of in epilepsy patients.

\section{Conflict of interest}

None of the authors has any conflicts of interest to disclose.

\section{Funds}

This work was supported by the science and technology research project of Henan Provincial Department of Education (grant number 202102310185) and special research project of Traditional Chinese Medicine in Henan Province (grant number 2019ZY2144).

\section{Acknowledgments}

Ming-Liang Zhang, Fang-Zhou Liu and Xiao-Long Chen conducted the literature search and study selection, performed data extraction and evaluated study quality. Wei-Xia Li, Xiao-Yan Wang and Hui Zhang verified quality assessments. Pan-Pan Chen and Wen-Hui Jia performed the quantitative meta-analyses and drafted the manuscript with contributions from the other authors. Lu Niu and Yan Wang helped in the interpretation of results. Jin-Fa Tang was responsible for the project and participated in its implementation. All authors read and approved the final manuscript.

\section{References}

1. C C, M P, F M, G M, M G, GGAM P, E C, S R. Interactions Between Antiepileptic and Antibiotic Drugs: A Systematic Review and Meta-Analysis with Dosing Implications. Clinical pharmacokinetics 2019; 58: $875-86$.

2. TN F, RJ B. The relationship between the pharmacology of antiepileptic drugs and human gene variation: an overview. Epilepsy \& behavior : E\&B 2005; 7: 18-36.

3. S G, M G-D, R B, S S, K B, M G, K N, B V, M G, K K, P T, H K, S G, A S, SK B, Consortium IGV, R K. Genetic profile of patients with epilepsy on first-line antiepileptic drugs and potential directions for personalized treatment. Pharmacogenomics 2010; 11: 927-41.

4. D T-S, A L, U F, J K. The clinical role of genetic polymorphisms in drug-metabolizing enzymes. The pharmacogenomics journal 2008; 8: 4-15.

5. BM K, KE T, CJ W, SM K, DL K, FJ G, RH L. Human liver carbamazepine metabolism. Role of CYP3A4 and CYP2C8 in 10,11-epoxide formation. Biochemical pharmacology 1994; 47: 1969-79.

6. CF T, SG L, J K, JS L, DJ M, TE K, RB A. PharmGKB summary: carbamazepine pathway. Pharmacogenetics and genomics 2011; 21: 906-10.

7. Chbili C, Fathallah N, Laouani A, Nouira M, Hassine A, Ben Amor S, Ben Ammou S, Ben Salem C, Saguem S. Effects of EPHX1 and CYP3A4*22 genetic polymorphisms on carbamazepine metabolism and drug response among Tunisian epileptic patients. Journal of neurogenetics 2016; 30: 16-21. 
8. Daci A, Beretta G, Vllasaliu D, Shala A, Govori V, Norata GD, Krasniqi S. Polymorphic Variants of SCN1A and EPHX1 Influence Plasma Carbamazepine Concentration, Metabolism and Pharmacoresistance in a Population of Kosovar Albanian Epileptic Patients. PloS one 2015; 10: e0142408.

9. H P, M F, W L. P-Glycoprotein-mediated efflux of phenobarbital, lamotrigine, and felbamate at the blood-brain barrier: evidence from microdialysis experiments in rats. Neuroscience letters 2002; 327: 173-6.

10. H P, M F, W L. P-glycoprotein and multidrug resistance-associated protein are involved in the regulation of extracellular levels of the major antiepileptic drug carbamazepine in the brain. Neuroreport 2001; 12: 3557-60.

11. AJ B-T. MDR1 (ABCB1) polymorphisms: functional effects and clinical implications. Revista de investigacion clinica; organo del Hospital de Enfermedades de la Nutricion 2013; 65: 445-54.

12. L B, BS H, HK N, VC W, PW N, CH L, NC S, C Z, B T, GW W, HJ T, AA R, Z M, P K. Case-control association study of polymorphisms in the voltage-gated sodium channel genes SCN1A, SCN2A, SCN3A, SCN1B, and SCN2B and epilepsy. Human genetics 2014; 133: 651-9.

13. Nazish HR, Ali N, Ullah S. The possible effect of SCN1A and SCN2A genetic variants on carbamazepine response among Khyber Pakhtunkhwa epileptic patients, Pakistan. Therapeutics and clinical risk management 2018; 14: 2305-13.

14. Abe T, Seo T, Ishitsu T, Nakagawa T, Hori M, Nakagawa K. Association between SCN1A polymorphism and carbamazepine-resistant epilepsy. British journal of clinical pharmacology 2008; 66: 304-7.

15. GX Z, ML S, Z Z, P W, CX X, GH H. Association between EPHX1 polymorphisms and carbamazepine metabolism in epilepsy: a meta-analysis. International journal of clinical pharmacy 2019; 41: 1414-28.

16. Hung CC, Chang WL, Ho JL, Tai JJ, Hsieh TJ, Huang HC, Hsieh YW, Liou HH. Association of polymorphisms in EPHX1, UGT2B7, ABCB1, ABCC2, SCN1A and SCN2A genes with carbamazepine therapy optimization. Pharmacogenomics 2012; 13: 159-69.

17. Ma CL, Jiao Z, Wu XY, Hong Z, Wu ZY, Zhong MK. Association between PK/PD-involved gene polymorphisms and carbamazepine-individualized therapy. Pharmacogenomics 2015; 16: 1499-512.

18. Moher D, Liberati A, Tetzlaff J, Altman DG, The PG. Preferred Reporting Items for Systematic Reviews and Meta-Analyses: The PRISMA Statement. PLOS Medicine 2009; 6: e1000097.

19. Kwan P, Arzimanoglou A, Berg AT, Brodie MJ, Allen Hauser W, Mathern G, Moshé SL, Perucca E, Wiebe S, French J. Definition of drug resistant epilepsy: consensus proposal by the ad hoc Task Force of the ILAE Commission on Therapeutic Strategies. Epilepsia 2010; 51: 1069-77.

20. CK L, D M, M L. Newcastle-Ottawa Scale: comparing reviewers' to authors' assessments. BMC medical research methodology 2014; 14: 45.

21. Subenthiran S, Abdullah NR, Muniandy PK, Joseph JP, Cheong KC, Ismail Z, Mohamed Z. G2677T polymorphism can predict treatment outcome of Malaysians with complex partial seizures being treated with Carbamazepine. Genetics and molecular research : GMR 2013; 12: 5937-44.

22. Subenthiran S, Abdullah NR, Joseph JP, Muniandy PK, Mok BT, Kee CC, Ismail Z, Mohamed Z. Linkage disequilibrium between polymorphisms of $\mathrm{ABCB} 1$ and $\mathrm{ABCC} 2$ to predict the treatment outcome of Malaysians with complex partial seizures on treatment with carbamazepine mono-therapy at the Kuala Lumpur Hospital. PloS one 2013; 8: e64827.

23. Huang, JM, Qian Z, Chen, HY, Huang, Q, Huang, L, Liu, GJ, Tang, XL. Association of single nucleotide polymorphisms of SCN1A gene with therapeutic effect of carbamazepine among ethnic Zhuang Chinese patients with epilepsy. Chinese Journal of Medical Genetics 2019; 36(3): 271-274. 
24. Wang, P, Zhou, QH, Sheng, YH, Tang, BS, Liu, SZ, and Zhou, BT. Association between two functional SNPs of $S C N 1 A$ gene and efficacy of carbamazepine monotherapy for focal seizures in Chinese Han epileptic patients. Journal of Central South University (medical) 2014 (05): 433-441.

25. Yun W, Zhang F, Hu C, Luo X, Xue P, Wang J, Ge Y, Meng H, Guo Y. Effects of EPHX1, SCN1A and CYP3A4 genetic polymorphisms on plasma carbamazepine concentrations and pharmacoresistance in Chinese patients with epilepsy. Epilepsy research 2013; 107: 231-7.

26. Zhou BT, Zhou QH, Yin JY, Li GL, Xu XJ, Qu J, Liu D, Zhou HH, Liu ZQ. Comprehensive analysis of the association of SCN1A gene polymorphisms with the retention rate of carbamazepine following monotherapy for new-onset focal seizures in the Chinese Han population. Clinical and experimental pharmacology \& physiology 2012; 39: 379-84.

27. Sterjev Z, Trencevska GK, Cvetkovska E, Petrov I, Kuzmanovski I, Ribarska JT, Nestorovska AK, Matevska N, Naumovska Z, Jolevska-Trajkovic S, Dimovski A, Suturkova L. The association of C3435T single-nucleotide polymorphism, Pgp-glycoprotein gene expression levels and carbamazepine maintenance dose in patients with epilepsy. Neuropsychiatric disease and treatment 2012; 8: 191-6.

28. Zhao, X, He, ZK, Zhang, J, Yang, Z. Whether the SCN1A IVS5N+5 G>A polymorphism of the SCN1A gene affects the responsiveness to the Ant-epileptic drug carbamazepine. Journal of pediatric pharmacy 2011; (01): 26-28.

29. Meng H, Guo G, Ren J, Zhou H, Ge Y, Guo Y. Effects of ABCB1 polymorphisms on plasma carbamazepine concentrations and pharmacoresistance in Chinese patients with epilepsy. Epilepsy \& behavior 2011; 21: $27-30$.

30. Ozgon GO, Bebek N, Gul G, Cine N. Association of MDR1 (C3435T) polymorphism and resistance to carbamazepine in epileptic patients from Turkey. European neurology 2008; 59: 67-70.

31. Zhu X, Yun W, Sun X, Qiu F, Zhao L, Guo Y. Effects of major transporter and metabolizing enzyme gene polymorphisms on carbamazepine metabolism in Chinese patients with epilepsy. Pharmacogenomics 2014; 15: 1867-79.

32. Puranik YG, Birnbaum AK, Marino SE, Ahmed G, Cloyd JC, Remmel RP, Leppik IE, Lamba JK. Association of carbamazepine major metabolism and transport pathway gene polymorphisms and pharmacokinetics in patients with epilepsy. Pharmacogenomics 2013; 14: 35-45.

33. Ru, JL, He, XJ, Qiu, F, Sun, YX, Zhao, LM, Zhu, X. Effects of ABCBl polymorphisms on plasma carbamazepine concentration. Chinese Journal of Hospital Pharmacy 2012; (11): 820-824.

34. L Q, Y F, W W, K M, Z Y. Development, validation and clinical application of an online-SPE-LCHRMS/MS for simultaneous quantification of phenobarbital, phenytoin, carbamazepine, and its active metabolite carbamazepine 10,11-epoxide. Talanta 2016; 158: 77-88.

35. L B, T T. Clinical pharmacokinetics and pharmacological effects of carbamazepine and carbamazepine10,11-epoxide. An update. Clinical pharmacokinetics 1986; 11: 177-98.

36. M R, P B, H C. Prediction of drug-drug interactions with carbamazepine-10,11-epoxide using a new in vitro assay for epoxide hydrolase inhibition. Xenobiotica; the fate of foreign compounds in biological systems 2016; 46: 1076-84.

37. M M-B, GJ S, N H, E B, EA W, MJ B. Genetic variants in microsomal epoxide hydrolase influence carbamazepine dosing. Clinical neuropharmacology 2009; 32: 205-12.

38. Nakajima Y, Saito Y, Shiseki K, Fukushima-Uesaka H, Hasegawa R, Ozawa S, Sugai K, Katoh M, Saitoh O, Ohnuma T, Kawai M, Ohtsuki T, Suzuki C, Minami N, Kimura H, Goto Y, Kamatani N, Kaniwa N, Sawada J. Haplotype structures of EPHX1 and their effects on the metabolism of carbamazepine-10,11epoxide in Japanese epileptic patients. European journal of clinical pharmacology 2005; 61: 25-34. 
39. NM B, T O, J P, P SdS, H B. Polyamine Modulation of Anticonvulsant Drug Response: A Potential Mechanism Contributing to Pharmacoresistance in Chronic Epilepsy. The Journal of neuroscience : the official journal of the Society for Neuroscience 2018; 38: 5596-605.

40. CH T, KM K, AL G. SCN1A splice variants exhibit divergent sensitivity to commonly used antiepileptic drugs. Epilepsia 2011; 52: 1000-9.

41. M T, J D, P S, A B-B. Hydrolytic stability of selected pharmaceuticals and their transformation products. Chemosphere 2019; 236: 124236.

\section{Hosted file}

Figures.docx available at https://authorea.com/users/323724/articles/452271-abcb1-c3435c-t-and-ephx1-c-416a-g-polymorphisms-influence-plasma-carbamazepine-concentrationmetabolism-and-pharmacoresistance-in-epileptic-patients

Hosted file

Tables.docx available at https://authorea.com/users/323724/articles/452271-abcb1-c3435c-t-and-ephx1-c-416a-g-polymorphisms-influence-plasma-carbamazepine-concentrationmetabolism-and-pharmacoresistance-in-epileptic-patients 